\title{
El papel moderador de la autoeficacia profesional entre situaciones de acoso laboral y la salud en una muestra multiocupacional
}

\author{
Mariano Meseguer*, María I. Soler y Mariano García-Izquierdo
}

Facultad de Psicología de la Universidad de Murcia (España)

\begin{abstract}
Resumen: La autoeficacia es una competencia personal que actúa frente a los estresores incrementando o disminuyendo el malestar psicológico que estos pueden generar; por su parte el acoso psicológico o mobbing es uno de los factores psicosociales del medio laboral más dañinos para la salud de los trabajadores. Utilizando como marco de referencia el modelo teórico de demandas-recursos (JD-R model), este trabajo tiene como objetivo principal analizar el papel moderador de la autoeficacia profesional entre el acoso laboral y la salud autopercibida en una muestra multiocupacional de 772 trabajadores (379 hombres y 383 mujeres). Se administró un protocolo que contenía la escala NAQ para la medida del acoso laboral, el cuestionario de salud GHQ-12 y la subescala de autoeficacia profesional del MBI. Como resultado destaca el papel moderador de la autoeficacia sobre la salud en situaciones de acoso psicológico. Se discuten los resultados de este trabajo, las limitaciones y sus posibles implicaciones en las organizaciones.

Palabras clave: Mobbing; acoso laboral; autoeficacia profesional; salud.
\end{abstract}

Title: The moderating role of professional self-efficacy in situations of workplace bullying and self-perceived health.

Abstract: Self-efficacy is a personal competence stressors acts against increasing or decreasing the psychological discomfort they may generate, meanwhile workplace bullying or mobbing is one of the psychosocial work environment factors more damaging to the health of workers. Using as a framework the theoretical JD-R, this paper's main objective is to analyze the moderating role of professional self-efficacy between workplace bullying and perceived health status in a multi-occupational sample of 772 workers (379 men and 383 women). An anonymous questionnaire to workers was administered in active-duty after analyzing the data provided by the scale of workplace mobbing NAQ, Questionnaire GHQ-12 for the evaluation of the health and professional self-efficacy of the MBI. The result highlights the moderating role of self-efficacy on health in workplace bullying situations. To these work results of and its possible implication in the organizations are discussed.

Keys words: Workplace bullying; mobbing; self-efficacy professional; wellbeing.

\section{Introducción}

En la actualidad, el acoso psicológico o mobbing es uno de los factores psicosociales de riesgo que supone un grave problema por la elevada prevalencia y la repercusión negativa en la salud de los trabajadores (Einarsen \& Mikkelsen, 2003; Matthiesen \& Einarsen, 2001; 2004; Lallukka, Rahkonen \& Lahelma, 2011; Meseguer, Soler, Sáez \& García-Izquierdo, 2007; 2008, Niedhammer, David, Degioanni, Drummond \& Philip, 2009; Sancini et al., 2012; Yildirim, 2009; Zapf, Escartín, Einarsen Hoel \& Vartia, 2010).

Se trata de un proceso en el cual una o varias personas son objeto de conductas hostiles por parte de compañeros, subordinados o superiores en su ámbito de trabajo. La manifestación de estos comportamientos debe tener como características que sean repetidos (por ejemplo, con una frecuencia semanal) y prolongados (seis meses al menos); otra particularidad es la dificultad que el trabajador objeto de la animosidad encuentra para defenderse, observándose siempre una clara diferencia de poder a favor del agresor (Einarsen, Hoel, Zapf \& Cooper, 2003). Las distintas conductas hostiles tienen lugar en el marco de una relación de trabajo, pero no responde a las necesidades de organización del mismo; el propósito es crear un entorno hostil o humillante que perturbe la vida laboral de la víctima, y supone tanto un atentado a la dignidad del trabajador como un riesgo para su salud.

A la hora de explicar las causas y las consecuencias del acoso psicológico en el trabajo la mayor parte de la investi-

* Dirección para correspondencia [Correspondence address]: Mariano Meseguer de Pedro. Facultad de Psicología. Campus Universitario de Espinardo. 30100 Espinardo, Murcia (España). E-mail: marianom@um.es gación se ha realizado desde el enfoque del estrés. El modelo de demandas-recursos (JD-R model) (Bakker, Demerouti, De Boer \& Schaufeli, 2003) ha mostrado su utilidad a la hora de puestos de trabajo exigen demandas que pueden considerarse estresores cuando requieren del trabajador un esfuerzo elevado y mantenido en el tiempo para conseguir un determinado nivel de rendimiento. Por otro lado, los recursos tienen la capacidad de atenuar las exigencias de las demandas; es decir, que pueden reducir la percepción de éstas y, por tanto, las respuestas negativas asociadas. En suma, el modelo JD-R propone que altas demandas de trabajo y una falta de recursos dará lugar a distintas manifestaciones de estrés. Siguiendo este modelo, Jourdain y Chênevert (2010) concluyen que para prevenir y controlar las consecuencias negativas (por ejemplo, del burnout) resulta necesario una doble estrategia: disminuir las demandas laborales e incrementar los recursos disponibles.

El hecho de que en situaciones de estrés laboral similares, unas personas aumenten sus niveles de estrés y otras no, se suele atribuir a las variables individuales (recursos) por lo que es de gran importancia analizar cuáles pueden actuar como mitigadores de las consecuencias en la salud de los trabajadores. La idea fundamental subyacente es que la salud puede diferir no sólo por las situaciones sino también por la intervención de variables personales. Sin embargo, mientras que las características organizativas y del puesto de trabajo han ocupado una buena parte de la investigación del acoso laboral, la mayoría de los determinantes individuales han sido tratados someramente, aunque recientemente se haya puesto cierta atención en ellos.

Así, por ejemplo, Einarsen et al. (2003) y Salin (2003) plantean que la percepción subjetiva de acoso resulta necesaexplicar el estrés laboral crónico. Básicamente, señala que los 
ria para comprender este fenómeno y entender por qué no todas las víctimas reaccionan de la misma forma. Matthiesen y Einarsen (2004) analizaron el papel de la afectividad negativa y positiva, y concluyeron que podían modular en la manifestación de síntomas psicosomáticos. De forma similar, Nielsen, Matthiesen y Einarsen (2008) indican que el sentido de la coherencia, disposición individual de percibir el ambiente como comprensible y manejable, es un protector de la salud en las víctimas de acoso. Igualmente, Vie, Glasø y Einarsen (2011) mostraron que el autoetiquetado, creencia que una persona tiene de ser efectivamente objeto de acoso y que le lleva a reconocerse como víctima, desempeña un papel moderador entre la exposición a las conductas de acoso y las consecuencias para la salud. En otro estudio posterior, estos mismos autores, mostraron que las emociones positivas y negativas son mediadores de la relación entre la exposición al acoso y los problemas musculoesqueléticos (Vie, Glasø y Einarsen, 2012). En resumen, los resultados de distintas investigaciones sugieren que las características individuales son importantes a la hora de reaccionar ante posibles situaciones de acoso laboral y pueden explicar, al menos parcialmente, los efectos sobre la salud.

En esta línea, Mikkelsen y Einarsen (2002) mostraron cómo la autoeficacia generalizada actuaba como moderador de la relación entre la exposición a situaciones de acoso y sus consecuencias para la salud. La autoeficacia, aspecto central de la Teoría Social Cognitiva, se define como "la creencia en las propias capacidades para organizar y ejecutar los cursos de acción necesarios para producir determinados logros o resultados" (Bandura, 1997, p. 3). Básicamente, las investigaciones han señalado que la autoeficacia es un recurso personal que ayuda a las personas a enfrentarse a situaciones exigentes y protege su salud (Lubbers, Loughlin \& Zweig, 2005; Parker, Jimmieson \& Johnson, 2011).

Como sugiere Bandura (1997), la autoeficacia puede estar determinada por su nivel de especificidad, y por tanto será más determinante cuanto más se ajuste a un dominio concreto. Así, la autoeficacia en el trabajo (o profesional) será más adecuada para realizar análisis en entornos laborales que la generalizada (Salanova, Grau \& Martínez, 2005). Desde el punto de vista de la teoría, esto es así porque las creencias de eficacia son específicas de un dominio concreto (Bandura, 2001). La investigación previa apoya la necesidad de utilizar medidas de autoeficacia específicas de ámbitos concretos ya que se encuentran resultados mucho más robustos (Bandura, 1997; Maffei, Spontón, Spontón, Castellano \& Medrano, 2012; Pepe, Farnese, Avalone \& Vecchione, 2010).

Atendiendo a la autoeficacia específica en el contexto laboral, se han observado algunos resultados interesantes. Por ejemplo, que determina la cantidad de intentos y el tiempo que invierten los trabajadores para resolver problemas (Salanova, Bresó \& Schaufeli, 2005); también afecta a las percepciones de control que las personas poseen sobre el entorno, moderando los efectos de estresores tales como la sobrecarga de trabajo, la rutina o la presión temporal (Salanova et al., 2005). También, pero en sentido contrario, elevadas creen- cias de autoeficacia profesional favorecen la dedicación y la satisfacción de los trabajadores (Salanova, Schaufeli, Llorens, Peiró \& Grau, 2000). En definitiva, la autoeficacia profesional actúa frente a los estresores disminuyendo o aumentando el malestar psicológico que estos pueden generar (Salanova, Grau, Cifré \& Llorens, 2000; Salanova et al., 2005).

En resumen, los efectos negativos sobre la salud provienen tanto de las características personales del trabajador como del ambiente laboral, y recientemente se está incidiendo en la importancia de incluir y centrar la investigación en los recursos personales de los trabajadores (Moreno-Jiménez, Rodríguez-Muñoz, Moreno \& Garrosa, 2006; Schaufeli \& Bakker, 2004). De acuerdo con lo anteriormente expuesto, el objetivo de este trabajo es doble: por un lado, analizar las relaciones entre la autoeficacia profesional, la exposición a conductas de acoso laboral y la salud; y por otro, examinar el papel moderador de la autoeficacia profesional entre la percepción de exposición a las conductas de hostigamiento y la salud.

En concreto, sobre la base del modelo $J D-R$ se postulan las siguientes hipótesis:

Hipótesis 1: la percepción de exposición a conductas de acoso se asociará negativamente con la salud.

Hipótesis 2: la autoeficacia profesional se asociará negativamente con la percepción de exposición a conductas de acoso laboral y positivamente con la salud.

Hipótesis 3: la autoeficacia profesional moderará la relación entre la percepción al acoso laboral y la salud. Así, los trabajadores con altas puntuaciones en autoeficacia referirán mejor salud en situaciones de alta exposición a conductas de acoso.

\section{Método}

\section{Participantes}

La muestra está compuesta por 772 trabajadores $(49.7 \%$ hombres y $50.3 \%$ mujeres), con una media de edad de 35.59 años $(D T=12.01$; rango $=18-63$ años $)$ y una experiencia profesional media de 9.10 años $(D T=10.15$; rango $=2$ meses-45 años). Atendiendo a la actividad de la empresa, la muestra se distribuye en comercio (22.1\%), industria $(15.4 \%)$, hostelería $(13.1 \%)$, administración pública $(8.8 \%)$, sanidad $(8.6 \%)$, construcción $(8.1 \%)$, educación $(7.5 \%)$, agricultura $(4.7 \%)$ y transporte $(3 \%)$, el resto no incluyó este dato $(6 \%)$. La mayoría de los participantes están casados o viven en pareja $(71 \%)$. Según el nivel de formación reglada, el $36.9 \%$ de la muestra poseen estudios universitarios, el $21.2 \%$ primarios, el 18.5\% Formación Profesional y el 17.7\% bachillerato. En cuanto al puesto de trabajo, el 54\% pertenecen a la categoría de personal de base, el $29.3 \%$ son técnicos y el $9.5 \%$ forman parte del grupo de directivos. El 64.6\% tienen contrato indefinido y el resto temporal. 


\section{Procedimiento}

La recogida de datos se realizó en el puesto de trabajo mediante un protocolo, que incluía tanto las instrucciones como el objetivo científico, y compuesto por varios cuestionarios de autoaplicación que fueron entregados a los trabajadores por miembros del equipo investigador; una vez completados, fueron introducidos en un sobre cerrado y recopilados entre enero y marzo de 2011. Todos los participantes lo hicieron voluntariamente, y se garantizó el anonimato y la confidencialidad. Se entregaron 1000 protocolos y fueron devueltos 885 , de los cuales se rechazaron 113 por no estar correctamente cumplimentados (tasa de respuesta $77.2 \%)$.

\section{Instrumentos}

Para la medida del acoso laboral se utilizó la escala de acoso NAQ de Einarsen y Raknes en la adaptación española de Soler, Meseguer, García-Izquierdo e Hidalgo (2010). Este cuestionario evalúa el acoso laboral percibido; para ello solicita a los participantes que respondan con qué frecuencia en los últimos 6 meses han percibido cada uno de las 24 conductas hostiles o "actos negativos" en el trabajo que se señalan. La forma de respuesta es una escala tipo Likert de 5 puntos, desde 1 (nunca) hasta 5 (diariamente). Un ejemplo de ítem es el 13, "se le recuerda persistentemente sus errores". Mostró una consistencia interna según el $\alpha$ de Cronbach de 89 .

Para evaluar la salud se utilizó el Cuestionario General de Salud (GHQ-12) de Goldberg y Williams en la adaptación española de Sánchez-López y Dresch (2008) de 12 ítems. Los participantes deben responder a las frases que se les presenta según una escala tipo Likert de 4 puntos desde 0 (no, en absoluto), hasta 3 (mucho más de lo habitual). Un ejemplo de ítem es el 2 " ¿sus preocupaciones le han hecho perder mucho el sueño?”. Mayores puntuaciones indican peor salud. Presentó una consistencia interna de $\alpha .76$.

La autoeficacia profesional se ha medido con la subescala del mismo nombre del Maslach Burnout Inventory General Survey (MBI-GS) de Schaufeli, Maslach, Leiter y Jackson (1996) en la versión española de Salanova et al. (2000). Contiene tres sub-escalas independientes: agotamiento emocional, cinismo y autoeficacia profesional. Esta última es la que ha sido utilizada y está conformada por 5 ítems que se refieren a la eficacia percibida en el desarrollo del trabajo (por ejemplo, el ítem 5: "puedo resolver de manera eficaz los problemas que surgen en mi trabajo"). Todos los ítems se valoran mediante una escala de siete puntos que va desde 0 (nunca) hasta 6 (siempre). La consistencia interna de la escala fue de $\alpha .87$.

\section{Análisis de los datos}

El tratamiento estadístico de los datos se ha realizado mediante el programa informático SPSS 19.0. En primer lu- gar, se realizaron los estadísticos descriptivos y de fiabilidad. En segundo lugar, el cálculo del análisis de regresión jerárquica siguiendo las recomendaciones de Baron y Kenny (1986) para el análisis de moderación. Por último, se aplicó la prueba de Sobel para la significación de efectos indirectos y probar el posible papel moderador de la autoeficacia profesional.

\section{Resultados}

La media, la desviación típica y las correlaciones entre las distintas variables del estudio se muestran en la tabla 1. Todas las correlaciones son significativas y en el sentido teórico esperado, excepto la edad con la autoeficacia profesional.

Tabla 1. Medias, desviaciones típicas y correlaciones.

\begin{tabular}{lccccc}
\hline Variables & $M$ & $D T$ & 1 & 2 & 3 \\
\hline 1. Edad & 35.60 & 12.01 & & & \\
2. Acoso Laboral & 1.29 & .39 & $-.09^{*}$ & & \\
3. Autoeficacia & 4.44 & .98 & .07 & $-.28^{* *}$ & \\
Profesional & & & & & \\
4. Salud & 1.82 & .46 & $-.13^{* *}$ & $.48^{* *}$ & $-.21^{* *}$ \\
\hline
\end{tabular}

4. Salud

Para probar la moderación, utilizamos el análisis de regresión jerárquica. Según Baron y Kenny (1986), la moderación existe si se resuelven los criterios siguientes: a) el predictor (acoso laboral) debe estar relacionado significativamente con la variable dependiente (salud) y con la variable moderadora (autoeficacia profesional). Este punto, puede observarse en la tabla 1, donde las correlaciones son significativas entre las tres variables; b) la variable moderadora (autoeficacia profesional) debe estar significativamente asociada con la variable dependiente (salud), que como muestra la tabla 1 presenta una correlación estadísticamente significativa $\left.\left(r_{x y}=-.21 ; p<.01\right) ; \mathrm{c}\right)$ el efecto del predictor sobre la variable dependiente desaparece o se reduce cuando participa el moderador en el modelo de predicción, como se puede observar en la tabla 2 , en el paso 3 del análisis de regresión se obtiene una variación de $\beta=.49 ; p<.001$ a $\beta=.47 ; p<.01$. La prueba de Sobel reveló que el efecto moderador de la autoeficacia profesional fue significativo en el aumento de la $R^{2}$ ( $p$ $<.05)$.

Tabla 2. Resultados del análisis de regresión jerárquica.

\begin{tabular}{llll}
\hline \multicolumn{4}{c}{ Salud } \\
\hline Variable Independiente & $\beta$ & $\mathrm{R}^{2}$ & $\Delta \mathrm{R}^{2}$ \\
\hline Paso 1 & .14 & .02 & $.02^{* *}$ \\
Edad & & & \\
Paso 2 & .18 & & \\
Edad & .49 & .26 & $.24 * *$ \\
Acoso Laboral & & & \\
Paso 3 & .19 & & \\
Edad & .47 & & \\
Acoso Laboral & -.26 & & \\
Autoeficacia & .19 & .27 & $.01 *$ \\
AutoeficaciaxAcoso & & & \\
* $^{*}<.05 ; * *<.01$ & & &
\end{tabular}


En resumen, de los datos se deduce que la autoeficacia profesional modera la relación entre la percepción de exposición a las conductas de acoso y la salud. Con el fin de analizar adecuadamente esta relación se realizó su representación gráfica (ver figura 1). Para determinar los valores de las variables se ha tomado $+/-1$ desviación típica respecto de la media.

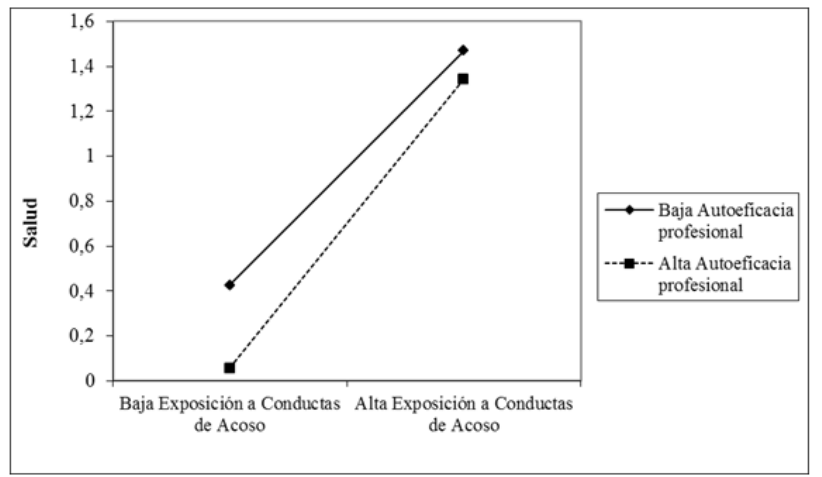

Figura 1. Interacción entre autoeficacia profesional y exposición a conduc tas de acoso laboral en la predicción de la salud (nótese que a mayor puntuación en el eje "salud" aumenta el malestar).

\section{Discusión}

El propósito de este trabajo fue indagar las relaciones entre la autoeficacia profesional, la percepción de exposición a conductas de acoso laboral y la salud en trabajadores. Los resultados corroboran las hipótesis planteadas. Las dos primeras, relativas a la existencia de relaciones significativas entre autoeficacia profesional, conductas de acoso y salud están en concordancia con los hallazgos de estudios previos (García-Izquierdo, García-Izquierdo \& Ramos-Villagrasa, 2007; Ríos, Sánchez-Meca \& Godoy, 2010).

Los resultados también confirman la tercera hipótesis de este trabajo que planteaba el papel moderador de la autoeficacia profesional. Este resultado es congruente con el modelo $J D-R$, de manera que los trabajadores tienen capacidades (recursos) que pueden activar como fuente de resistencia ante los acontecimientos estresantes (Baillien, De Cuyper \& De Witte, 2011; Hunter, Mora \& Ortega, 2004).Igualmente, los datos están en la línea de la Teoría Social Cognitiva cuando indica que las personas con elevados niveles de autoeficacia poseen confianza en sus habilidades para responder a los estímulos del medio y pueden ejercer cierto control sobre ellos (Bandura, 1997). Además, se pone de manifiesto el efecto de interacción entre demandas laborales (acoso laboral) y recursos personales (autoeficacia profesional), de manera que los trabajadores con altos niveles de autoeficacia profesional aumentarán sus conductas de afrontamiento positivas en situaciones de estrés laboral (Domenech \& Gómez, 2010; Salanova et al., 2005; Sánchez-Cardona et al., 2012). Los resultados obtenidos también se ven apoyados por otros estudios donde la autoeficacia profesional ha mostrado su relevancia como variable moderadora en los procesos de estrés y salud (Jex \& Bliese, 1999; Lu, Siu \& Cooper, 2005; Sonnentag \& Spychala, 2012).

Como se ha comentado con anterioridad, desde la propuesta de la Teoría Social Cognitiva un elemento básico de la autoeficacia procede de la creencia en el control sobre los elementos del entorno de la persona. Sin embargo, una característica de los procesos de acoso laboral es que el trabajador se siente cada vez más indefenso y, por tanto, con menos capacidad de controlar el entorno laboral, reduciendo así sus posibilidades de resolver eficazmente las situaciones problemáticas que se le presentan. Por ejemplo, tanto de forma directa como indirecta, se le dificulta la comunicación con compañeros o superiores, pierde posibilidades de apoyo social en el trabajo, se le encomiendan actividades por debajo de su nivel de cualificación, se le recuerdan persistentemente sus errores, etc., (Rodríguez-Caballeira, Escartín, Visauta, Porrúa \& Martín-Peña, 2010). Así, tanto desde el punto de vista profesional como personal, cuando el acoso se generaliza, el propio proceso tiende a eliminar cualquier respuesta funcional de la víctima, lo que incrementa el desequilibrio entre acosador y acosado.

De ahí, el gran interés que puede tener esta investigación, cómo un trabajador puede mantener una creencia en sus capacidades profesionales en un entorno tan hostil, y si esa creencia mantendría los niveles de salud y bienestar. Es destacable señalar que el papel de la autoeficacia profesional es similar al del resto de variables personales analizadas hasta ahora en la investigación con respecto al acoso (afectividad negativa, sentido de la coherencia, autoetiquetado, autoeficacia generalizada o emociones positivas), en los niveles bajos de percepción de conductas hostiles, las variables personales desempeñan un papel moderador, reduciendo los niveles de malestar de forma significativa $\mathrm{y}$, aunque en niveles altos, cuando se entra en la espiral del conflicto, también reduce la percepción sobre el propio estado de salud, ese papel amortiguador va disminuyendo. Posiblemente, sería de gran interés que en posteriores investigaciones se explorase el papel que otras variables personales pueden tener en el desarrollo del acoso laboral, por ejemplo, la resiliencia, el optimismo o la esperanza.

\section{Limitaciones}

El estudio contiene algunas limitaciones que conviene señalar. En primer lugar, los datos recogidos provienen de pruebas de autoinforme y el diseño es transversal. La utilización de cuestionarios puede provocar un sesgo en la respuesta de los participantes, exacerbar la varianza común y aumentar artificialmente las correlaciones entre variables. Por otro lado, es evidente que la evaluación de la percepción de acoso mediante cuestionarios aporta una información relevante que ha sido utilizada para la estimación de la prevalencia, las conductas implicadas, la procedencia del acoso y la asociación con variables antecedentes y consecuentes (Cowie, Naylor, Rivers, Smith \& Pereira, 2002). Sin embar- 
go, no se está midiendo la presencia objetiva de acoso laboral, si no la percepción de estar expuesto a distintas conductas que conforman este fenómeno. Sin duda, esta es una cuestión aun por resolver, a pesar de que es primordial para el desarrollo de estrategias de intervención y de prevención. Por ello, la mejora de la evaluación del acoso debe proseguir, y la utilización de diferentes métodos y perspectivas parece necesaria para lograr un conocimiento profundo del proceso (Cowie et al., 2002).

La segunda limitación proviene del hecho de que en los resultados haya podido intervenir alguna otra variable personal que puede tener efectos en la predicción de la salud y que no haya sido contemplada. Por lo tanto, son necesarios estudios que incluyan distintas variables personales relevantes. Por último, los datos se refieren a una muestra heterogénea de trabajadores; esta limitación sugiere que futuras investigaciones se realicen con distintos grupos profesionales específicos.

\section{Referencias}

Baillien, E., De Cuyper, N., \& De Witte, H. (2011). Job autonomy and workload as antecedents of workplace bullying: A two-wave test of Karasek's Job Demand Control Model for targets and perpetrators. Journal of occupational and Organizational Psychology, 84(1), 191-208. doi: 10.1348/096317910X508371.

Bandura, A. (1997). Self-efficacy: Toward a unifying theory of behavioral change. Psychological Review, 84(2), 191-215. doi: 10.1037/0033295X.84.2.191.

Bandura, A. (2001). Social cognitive theory: an agentic perspective. Annual Review of Psychology, 52, 1-26.

Bakker, A. B., Demerouti, E., De Boer, E., \& Schaufeli, W. B. (2003). Job demands and job resources as predictors of absence duration and frequency. Journal of Vocational Behavior, 62, 341-356. doi:10.1016/S00018791(02)00030-1.

Baron, R. M. \& Kenny, D. A. (1986). The moderator-mediator variable distinction in social psychological research: Conceptual, strategic and statistical considerations. Journal of Personality and Social Psychology, 51, 11731182. doi.org/10.1037/0022-3514.51.6.1173.

Cowie, H., Naylor, P., Rivers, I., Smith, P. K. \& Pereira, B. (2002). Measuring workplace bullying. Aggression and Violent Behavior, 7, 33-51. doi.org/10.1016/S1359-1789(00)00034-3.

Domenech, F. \& Gómez, A. (2010). Barriers perceived by teachers at work, coping strategies, self-efficacy and burnout. The Spanish Journal of Psychology, 13(2), 637-654.

Einarsen, S., Hoel, H., Zapf, D. \& Cooper, C. L. (2003). The concept of bullying at work. In Einarsen, S., Hoel, H., Zapf, D. \& Cooper, C. L. (Eds), Bullying and Emotional Abuse in the Workplace. International perspectives in research and practice (pp. 3-30). London: Taylor \& Francis.

Einarsen, S., \& Mikkelsen, E. G. (2003). Individual effects of exposure to bullying at work. In Einarsen, S., Hoel, H., Zapf, D. \& Cooper C. L. (Eds.), Bullying and emotional abuse in the workplace. International perspectives in research and practice (pp. 127-144). London: Taylor \& Francis.

García-Izquierdo, A. L., García-Izquierdo, M. \& Ramos-Villagrasa, P. J. (2007). Aportaciones de la inteligencia emocional y la autoeficacia: Aplicaciones para la selección de personal. Anales de Psicología, 23(2), 231239.

Hunter, H., Mora, J. \& Ortega, R. (2004).The long-term effects of coping strategy use in victims of bullying. The Spanish Journal of Psychology, 7(1), 3-12.

\section{Implicaciones}

La primera implicación deriva del hecho de que el acoso laboral tiene un gran impacto en la salud de quienes lo sufren, y merece ser considerado por las organizaciones como situaciones indeseables y que se apliquen con rigor las distintas medidas preventivas para su erradicación. En segundo lugar, el fomento de la autoeficacia profesional mitigaría las consecuencias de las conductas de acoso, aumentarían las estrategias activas de los distintos empleados para la resolución de problemas interpersonales.

En definitiva, una mejora salud depende de la eliminación, control y modificación de los estresores laborales. Sin embargo, esto no es suficiente, se debe atender también al desarrollo de los recursos personales de los trabajadores. Así, el fomento de la autoeficacia profesional mediante acciones formativas específicas ayudaría a paliar los efectos negativos de distintos estresores psicosociales como puede ser el acoso laboral.

Jex, S. M. \& Bliese, P. D. (1999). Efficacy beliefs as a moderator of the impact of work-related stressors: a multilevel study. Journal of Applied Psychology, 84, 349-361. doi: org/10.1037//0021-9010.84.3.349.

Jourdain, G. \& Chênevert, D. (2010). Job demands-resources, burnout and intention to leave the nursing profession: A questionnaire study. International Journal of Nursing Studies, 47(6), 709-722. doi: 10.1016/j.ijnurstu.2009.11.007.

Lallukka, T., Rahkonen, O. \& Lahelma, E. (2011). Workplace bullying and subsequent sleep problems--the Helsinki Health Study. Scandinavian journal of work, environment \& health, 37(3), 204.

Lu, C. Q., Siu, O. L. \& Cooper, C. L. (2005). Managers' occupational stress in China: The role of managerial self-efficacy. Personality and Individual Differences, 38, 569-578. doi: 10.1016/j.paid.2004.05.012.

Lubbers, R., Loughlin, C. \& Zweig, D. (2005). Young workers' job selfefficacy and affect: Pathways to health and performance. Journal of Vocational Behavior, 67(2), 199-214. doi: org/10.1016/j.jvb.2004.03.002.

Maffei, L., Spontón, C., Spontón, M., Castellano, E., \& Medrano, L. A. (2012). Adaptación del Cuestionario de Autoeficacia Profesional (AU10) a la población de trabajadores cordobeses. Pensamiento Psicológico, 10(1), 51-62.

Matthiesen, S. B. \& Einarsen, S. (2001). MMPI-2 configurations among victims of bulling at work. European Journal of Work and Organizational Psychology, 10, 467-484. doi: 10.1080/13594320143000753.

Matthiesen, S. B. \& Einarsen, S. (2004). Psychiatric distress and symptoms of PTSD among victims of bullying at work. British Journal of Guidance and Counseling, 32, 335-356. doi: 10.1080/03069880410001723558.

Meseguer, M., Soler M. I., Sáez, M. C. \& García-Izquierdo, M. (2008). Workplace mobbing and effects on workers health. The Spanish Journal of Psychology, 11(1), 219-227.

Meseguer, M., Soler M. I., Sáez, M. C. \& García-Izquierdo, M. (2008). Incidencia, componentes y origen del mobbing en el trabajo en el sector hortofrutícola. Anales de Psicología, 23(1), 92-100.

Mikkelsen, E. G. \& Einarsen, S. (2002). Relationships between exposure to bullying at work and psychological and psychosomatic health complaints: The role of state negative affectivity and generalized selfefficacy. Scandinavian Journal of Psychology, 43, 397-405. doi: 10.1111/1467-9450.00307.

Moreno-Jiménez, B., Rodríguez-Muñoz, A., Moreno, Y. \& Garrosa, E. (2006). El papel moderador de la asertividad y la ansiedad social en el acoso psicológico en el trabajo: dos estudios empíricos. Revista de Psicología del Trabajo y de las Organizaciones, 22(3), 363-380. 
Niedhammer, I., David, S., Degioanni, S., Drummond, A., \& Philip, P. (2009). Workplace bullying and sleep disturbances: findings from a large scale cross-sectional survey in the French working population. Sleep, 32(9), 1211.

Nielsen, M. B., Matthiesen, S. B. \& Einarsen, S. (2008). Sense of coherence as a protective mechanism among targets of workplace bullying. Journal of Occupational Health Psychology, 13, 128-136. doi: 10.1037/10768998.13.2.128.

Parker, S. L., Jimmieson, N. L. \& Johnson, K. M. (2011). General selfefficacy influences affective task reactions during a work simulation: the temporal effects of changes in workload at different levels of control. Anxiety Stress Coping, 26(2), 217-39. doi: 10.1080/10615806.2011.651616.

Pepe, S., Farnese, M. L., Avalone, F., \& Vecchione, M. (2010). Work selfefficacy scale and search for work self-efficacy scale: a validation study in Spanish and Italian cultural contextes. Revista de Psicología del Trabajo y de las Organizaciones, 26(3), 201-210.

Ríos, M. I., Sánchez-Meca, J. \& Godoy, C. (2010). Personalidad resistente, autoeficacia y estado general de salud en profesionales de Enfermería de cuidados intensivos y urgencias. Psicothema, 22 (4), 600-605.

Rodríguez-Carballeira, A., Escartín, J., Visauta, B., Porrúa, C. \& MartínPeña, J. (2010). Categorization and Hierarchy of Workplace Bullying Strategies: A Delphi Survey. The Spanish Journal of Psychology, 13(1), $297-$ 308.

Salanova, M., Bresó, E. \& Schaufeli, W. B. (2005). Hacia un modelo de las creencias de eficacia en el estudio del burnout y del engagement. Ansiedad y Estrés, 11(2-3), 215-231.

Salanova, M., Grau, R., Cifré, E. \& Llorens, S. (2000). Computer training, frequency of use and burnout: the moderating role of computer selfefficacy. Computers in Human Behaviour, 16, 575-590. doi: 10.1016/S07475632(00)00028-5.

Salanova, M., Grau, R. M. \& Martínez, I. M. (2005). Demandas laborales y conductas de afrontamiento: el rol modulador de la autoeficacia profesional. Psicothema, 17(3), 390-395.

Salanova, M., Schaufeli, W. B., Llorens, S., Peiró, J. M. \& Grau, R. (2000). Desde el burnout al engagement: ¿Una nueva perspectiva? Revista de Psicología del Trabajo y de las Organizaciones, 16,117-134.

Salin, D (2003). Bullying and organisational politics in competitive and rapidly changing work environments. International Journal of Management and Decision Making: Workplace bullying, 4(1), 35-46.
Sánchez-Cardona, I., Rodriguez-Montalbán, R., Acevedo-Soto, E., Lugo, K. N., Torres-Oquendo, F. \& Toro-Alfonso, J. (2012). Self-Efficacy and Openness to Experience as Antecedent of Study Engagement: An Exploratory Analysis. Procedia-Social and Behavioral Sciences, 46, 2163-2167. doi: $\operatorname{org} / 10.1016 /$ j.sbspro.2012.05.446.

Sancini, A., Fioravanti, M., Andreozzi, G., Di Giorgio, V., Tomei, G., Tomei, F. \& Ciarrocca, M. (2012). Meta-analysis of studies examining long-term construction injury rates. Occupational Medicine, 62(5), 356-361. doi: 10.1093/occmed/kqs064.

Sánchez-López, M. P. \& Dresch, V. (2008). The 12-Item General Health Questionnaire (GHQ-12): Reliability, external validity and factor structure in the Spanish population. Psicothema, 20(4), 839-843.

Schaufeli, W. B., Maslach, C., Leiter, M. P. \& Jackson, S. E. (1996). Maslach Burnout Inventory-General Survey. In Maslach, C., Jackson, S.E. \& Leiter, M. P. (Eds.), The Maslach Burnout Inventory (3rd.ed)-Test Manual (pp. 19-26). Palo Alto: Consulting Psychologists Press.

Schaufeli, W. B. \& Bakker, A. B. (2004). Job demands, job resources and their relationship with burnoutand engagement: A multi-sample study. Journal of Organizational Behavior, 25, 293-315. doi: 10.1002/job.248.

Sonnentag, S. \& Spychala, A. (2012). Job Control and Job Stressors as Predictors of Proactive Work Behavior: Is Role Breadth Self-Efficacy the Link? Human Performance, 25(5), 412-431. doi: 10.1080/08959285.2012.721830.

Soler, M. I, Meseguer, M., García-Izquierdo, M. \& Hidalgo, M. D. (2010). Validez del cuestionario de conductas de hostigamiento (mobbing) de Einarsen y Raknes en una muestra del sector hortofrutícola. Ansiedad Estrés, 16(2-3), 151-162.

Vie, T. L., Glasø, L. \& Einaren, S. (2011) Health outcomes and self-labeling as a victim of workplace bullying. Journal of Psychosomatic Research, 70(1), 37-43. doi: 10.1016/j.jpsychores.2010.06.007.

Vie, T. L., Glasø, L., \& Einarsen, S. (2012). How does it feel? Workplace bullying, emotions and musculoskeletal complaints. Scandinavian Journal of Psychology, 153, 168-173. doi: 10.1111/j.1467-9450.2011.00932.x.

Yildirim, D. (2009). Bullying among nurses and its effects. International Nursing Review, 56(4), 504-511. doi: 10.1111/j.1466-7657.2009.00745.x.

Zapf, D., Escartín, J., Einarsen, S., Hoel, H. \& Vartia, M. (2010). Empirical findings on prevalence and risk groups of bullying in the workplace. Bullying and harassment in the workplace: Developments in theory, research, and practice, $75-105$.

(Articulo recibido: 22-10-2012; revisado: 09-5-2013, aceptado: 9-5-2013) 